\title{
Article \\ Study of 5G-NR-MIMO Links in the Presence of an Interferer
}

\author{
Avner Elgam *, Yael Balal (1) and Yosef Pinhasi \\ Faculty of Engineering, Ariel University, Ariel 40700, Israel; yaelba@ariel.ac.il (Y.B.); yosip@ariel.ac.il (Y.P.) \\ * Correspondence: avner.elgam@msmail.ariel.ac.il
}

Citation: Elgam, A.; Balal, Y.; Pinhasi, Y. Study of 5G-NR-MIMO Links in the Presence of an Interferer. Electronics 2021, 10, 732. https:// doi.org/10.3390/electronics10060732

Academic Editors: Ana Vázquez Alejos, Manuel García Sanchez, Francisco Falcone and Leyre Azpilicueta

Received: 27 February 2021

Accepted: 17 March 2021

Published: 19 March 2021

Publisher's Note: MDPI stays neutral with regard to jurisdictional claims in published maps and institutional affiliations.

Copyright: (C) 2021 by the authors. Licensee MDPI, Basel, Switzerland. This article is an open access article distributed under the terms and conditions of the Creative Commons Attribution (CC BY) license (https:// creativecommons.org/licenses/by/ $4.0 /)$.

\begin{abstract}
Many communication systems are based on the Multiple Input, Multiple Output (MIMO) scheme, and Orthogonal Space-time Block Transmit diversity Coding (OSTBC), combined with Maximal Ratio Receive Combining (MRRC), to create an optimal diversity system. A system with optimal diversity fixes and optimizes the channel's effects under multi-path and Rayleigh fading with maximum energy efficiency; however, the challenge does not end with dealing with the channel destruction of the multi-path impacts. Susceptibility to interference is a significant vulnerability in future wireless mobile networks. The 5th Generation New Radio (5G-NR) technologies bring hundreds of small cells and pieces of User Equipment (UE) per indoor or outdoor local area scenario under a specific Long Term Evolution (LTE)-based station (e-NodeB), or under 5G-NR base-station (g-NodeB). It is necessary to study issues that deal with many interference signals, and smart jammers from advanced communication equipment cause deterioration in the links between the UE, the small cells, and the NodeB. In this paper, we study and present the significant impact and performances of $2 \times 2$ Alamouti Phase-Shift Keying (PSK) modulation techniques in the presence of an interferer and a smart jammer. The destructive effects affecting the MIMO array and the advanced diversity technique without closed-loop MIMO are analyzed. The performance is evaluated in terms of Bit Error Rate (BER) vs. Signal to Interference Ratio (SIR). In addition, we proved the impairment of the orthogonal spectrum assumption mathematically.
\end{abstract}

Keywords: interferer; 5G-NR-MIMO; Alamouti

\section{Introduction}

The road map to advanced technologies such as Long Term Evolution (LTE) release 8-14 [1] and The 5th Generation New Radio (5G-NR) technology [2] have led to significant breakthroughs. Many studies and researchers have created a scientific basis for engineering challenges and physical problems, such as peak spectral efficiency, fast fading channel response, and destructive effects in a multi-path channel [3-6]. Dealing with fading and selective effects, a delay spread, high Doppler effects, fluctuation channels characterized in the time and frequency domains, and random scatters near the User Equipment (UE) or the small-cell are not trivial issues [4,5]. In addition, the demands in the real world of next-generation mobile technology for high mobility, low latency, spectrum efficiency, peak data rates, and ultra multi-user experience, with emphasis on multiple users and sharing radio access, are increasing and have expanded the research field. There are a number of threshold conditions that must be met in order to operate multi-user access techniques such as Orthogonal Multiple Access (OMA)/Non-OMA (NOMA) techniques [7-9], or Multi-User (MU)- Multiple Input, Multiple Output (MIMO) [10-12]. The first condition is the ability to decoding a specific stream transmission, from spatial-multiplexing streams, under a random Signal Interference Noise Ratio (SINR) with random interference signals. The second condition is to combines unlicensed and licensed technology. The meaning of this combination is to manage a significant number of the wireless device under specific macro cell, without sharing any Physical Upload Shared Channel (PUSCH), Without sharing a common scheduler, and also without any Demodulation Reference-Signals (DM-RS), which help to run algorithms for estimating and offsetting interfering, like 
Minimum Mean Square Error-Interference Rejection Combining (MMSE-IRC) [13-15]. For example, the use License Assisted Access (LAA) protocol [16,17], or Device-To-Device (DTD) technique [18], to get a significant channel capacity, in an environment with multiinterferences. Complicated subjects, such as radio management and fairness access, which require peak data in real-time applications, and also emerging applications such as intervehicle communication [19], increase these engineering challenges.

Previous works such as [20-22] have expanded this research area. These situations occur, especially in bands below $6 \mathrm{GHz}$, because spatial selectivity capacity requires instant time or frequency selectivity. The potential risk of exposure of essential international infrastructure in the private market or the military sector to a jammer attack or a multiinterference environment is troublesome.

On the one hand, there is massive investment in information security networks and core layers against cyber attacks. On the other hand, with some simple jammer devices, it is possible to cut off radio links between Internet of Things (IoT) devices inside medical refrigerators in hospitals or to disrupt the communication of GPS location systems [23]. They can also jam vehicle-to-vehicle communication between autonomous cars [24] and other critical systems. Military systems are also vulnerable to many jammer techniques, and the cumulative damage can be devastating.

In practice, we must develop and utilize two critical and advanced techniques when designing modern communication systems. First, an MIMO array or a massive MIMO technique is needed $[25,26]$. Second, smart control systems under Channel State Information (CSI) are known to the receiver and transmitter at the same time, streamed through the remote control channel, or the Physical Upload Control Channel (PUCCH). This capability will allow us to configure a system with flexible immunity, which includes a trade-off between handling the jammer and handling the strong interference signals in a multiinterference environment, and in parallel to create a large number of receive paths that are orthogonal to each other. This is needed for independent decoding and to increase the capacity of the channel. The benefits of this agenda are far-reaching. In a situation without any jammer or any interference signals between the transmitter and the receiver, we can achieve improvements over spatial multiplexing (SM) [27], or eigenvalue beamforming $[27,28]$. The above trade-off is expressed especially under the assumption of dynamic and random channel response or very high mobility of the transmitter relative to the receiver, or vice versa. Assuming a scattering-rich propagation channel or a two-ring model scatter environment [5] also creates a challenge. Improvements in channel estimation or estimation of the random Direction Of Arrival (DOA) of the receive paths [29] are also considered to produce improvement in this agenda.

Here, we present the significant effects of a smart jammer and interference on a wireless mobile network's physical (PHY) layer and in an unlicensed wireless communication environment. An understanding of these effects, without a doubt, will contribute to the modern techniques that we have mentioned. The common base of these phenomena is the destruction of the principle orthogonality region, which we expand in this paper (Appendix A). Orthogonality between the various receive paths in the surrounding multipath channels is particularly significant in the MIMO array with multiple receive and transmit antennas [27]; however, it allows for high vulnerability in MIMO techniques.

By creating orthogonality between the paths, we achieve maximum capacity in the channel [27]. The ability to receive multiple independent data streams fixes the internecine effects on the channel matrix, achieves a full rank in this matrix, and finds the optimum eigenvalue distribution that leads to a maximum capacity [27]. The maximum capacity is obtained when the ratio of the maximum eigenvalue to the minimum eigenvalue converges to 1 [27]. A system with only CSI at the Receiver (CSIR), every channel path has a power gain equal to the eigenvalue of the channel matrix. This is in addition to the fact that the effective transmit power is $\frac{1}{N_{t}}$ times the total transmit power, when $N_{t}$ is the number of transmit antennas. The orthogonality between the paths also leads to significant Success Interference Cancellation (SIC) implemented in many decoder techniques, especially in 
MRRC. In a multi-interfering environment there is damage to the orthogonal spectrum (Appendix A), and as a result, the performance of the receiver decreases significantly.

Another significant effect that we present in this paper adds to the orthogonality region's hit: replicating the interference and information symbols in the MRRC combiner after the interferer is received. This phenomenon is reflected in the constellation receiver, dependent on the Signal Interference Ratio (SIR), and the constellation order. Without a feedback system that fixes those duplicate symbols, this phenomenon will be complicated for advanced decoder capabilities such as Sphere Decoder (SD), Zero Forcings (ZF), and Best Linear Estimation (BLE). These effects intensify under the assumptions we make in this paper.

A clear example of our assumption can be found in the HetNet scenario [30,31]. In this case, we assume that we have several small cells and pieces of User Equipment (UE), both equipped with multi-transmission and multi-receiver antennas and communicated with a single macro-cell in the same network environment without sharing the same scheduler. In this case, we assume a full-duplex (FD) mode and Time Division Duplex (TDD) access through a slow-fading channel, and a random DOA receives angle, in addition to Uniform Linear Array (ULA) antennas. This situation [31] is complicated because the UE, the small cells, and the base-station can play the role of the transmitter, receiver, and interferer at the same time. Another assumption is that the transmitter and the interference's transmission are transmitted under the Orthogonal Space-Time Block Codes (OSTBC)-Alamouti scheme, also receive Alamouti decoder MRRC combiner with Maximum Likelihood (ML) search. The modulation is a Common Envelope (CE), to save energy and lower the Peak-to-Average Power Ratio (PAPR). Another assumption is that the interference occurs regularly, meaning that the statistical average of the product between the transmitter's symbols and the symbols of the receiver is different from zero [32].

In a military communication situation, Partial Band Noise (PBN) jammers [33], and smart jammers [21] are present. In these situations, a smart jammer learns and estimates the channel matrix between a legitimate transmission and his receiver. If we have a wide-band noise jammer [33] or even a PBN jammer [34], we can use the eigenvalue beamforming technique [35] and cancel the jammer effects with the null steering that eigenvalue beamforming will create [27]. In the smart jammer case, we must also control the MIMO array in the transmitter and in the receiver together, meaning also the CSI at the transmitter (CSIT).

In recent years, significantly advanced interference cancellation techniques, such as MMSE-Interference Rejection Combining (MMSE-IRC) and MMSE-Maximal Ratio Combining (MMSE-MRC), have been developed and implemented in advanced LTE [15,36,37]. The next step is the new algorithm interference, and anti-jamming generation is required, especially under the assumption of TDD access and Ultra-Reliable and Low-Latency Communications (URLLC) in 5G requirements. These requirements are a binding reduction in the computing of the co-variance [36] matrix and the time estimation of the channel, on the transmitter and the receiver sides. In our future works, we will address these requirements in developing fitting algorithms.

The purpose of this paper was to refine the challenge in order to deal with those impacts. We start by understanding the limitations of advanced diversity techniques as the OSTBC-Alamouti scheme, based only on the CSIR, without any closed loop-feedback. This article highlights the lack of re-feeding by focusing on the effects required for correction with the help of a control system. This closed-loop feedback creates and sends feedback that fixes the destructive multi-path and interference effects. In addition, the time response is reduced, and the channel with smart and effective CSIT is learned. These ideas open a window for other researchers. This study presents the impacts of an interferer or a smart jammer synchronizing with UE or with a small cell. Another purpose of this study was to enrich the importance of CSI Interference's Management (CSI-IM) [30] at the UE, at the macro base-station, or the small cells, in the same time, similar to the HetNet scenario. 
In unlicensed wireless communication, we clarify the importance of CSI-IM in transmissions, in an interferer or smart-jammer environment scenario. We illustrate these effects on the basic principle of MIMO techniques based on an open loop. Another purpose was to show that selective space techniques, such as eigenvalue beamforming and a null spacing matrix that reduces the effects of signal interference, are not enough to deal with highly dynamic interference or smart jammer attacks in the space domain. The main target is to overcome fluctuation channels in the time and frequency domain amid jammer or interferer issues.

Our idea is to create more flexible scheduling in the next 5G-NR transceiver and in new radio access. This paper can be integrated with other papers regarding unique geometric scattering models for MIMO channels [4,5]. Some studies work around modern communication with jammers $[15,21,31,36]$. This paper and future studies will complement these papers and provide different points of view on this subject. We divide strategies handling multi-interference into two mechanisms. The first is called analog Radio Frequency (RF) interference cancellation, and the second is digital interference cancellation [30]. We focus here on digital interference received at the combiner process. It is a complicated phenomenon that requires deep research. We must also remain focused on significant targets in 5G-NR, e.g., control model antennas, array beam patterns, and flexible air interfaces, to evaluate the algorithm design's impact on RF link performance. In addition, we must develop and research mechanisms that create a trade-off between dealing with jammers or strong signal interferences and increasing the channel's capacity with minimal energy.

The remainder of this paper is organized as follows: in Section 2, we present the basic principles of diversity transmission using the Alamouti MIMO technique. In Section 3, we present different interferer techniques: a general interferer, a smart jammer, and a PBN jammer. In Section 4, we present the simulation results in Bit Error Rate (BER) vs. SIR. Section 5 summarizes and concludes the paper.

\section{Diversity Transmission Using Alamouti MIMO Technique}

Transmit diversity can be obtained by Space-Time Coding (STC) that is designed to achieve maximum spatial diversity. One simple space-time code is the Alamouti code, which is used in most MIMO systems today. This method's significant advantage over other techniques is that it doesn't require CSIT, which reduces complexity while achieving maximum spatial diversity. We present the effects of interfering with the Alamouti STC. To understand these effects in-depth, we will provide a short brief on the known $2 \times 2$ Alamouti STC technique [1]. Figure 1 shows a block diagram of the $2 \times 2$ Alamouti system case. The transmission consists of two transmission antennas, $T X_{1}$ and $T X_{2}$. These antennas transmit in the Alamouti STC technique and represent the UE. Next, the signal transmits through a MIMO channel model with four flat fading paths independent and uncorrelated assume. These four paths are represented by complex channel gains $h_{11}$, $h_{12}, h_{21}, h_{22}$. The indexes are the indexes of $T X$ and $R X$ antennas, respectively, as shown in Table 1 [1,27]. The receive block includes two receive antennas, $R X_{1}$ and $R X_{2}$, and an Alamouti space-time decoder combined with MRRC or diversity combining channel state estimation with ML decoding. This receive block is subject to the rules of the basestation (NodeB).

Table 1. Definition of complex channel gain between the transmit and receive antennas.

\begin{tabular}{ccc}
\hline & $\boldsymbol{R} \boldsymbol{X}_{\mathbf{1}}$ & $\boldsymbol{R} \boldsymbol{X}_{\mathbf{2}}$ \\
\hline$T X_{1}$ & $h_{11}$ & $h_{12}$ \\
$T X_{2}$ & $h_{21}$ & $h_{22}$ \\
\hline
\end{tabular}




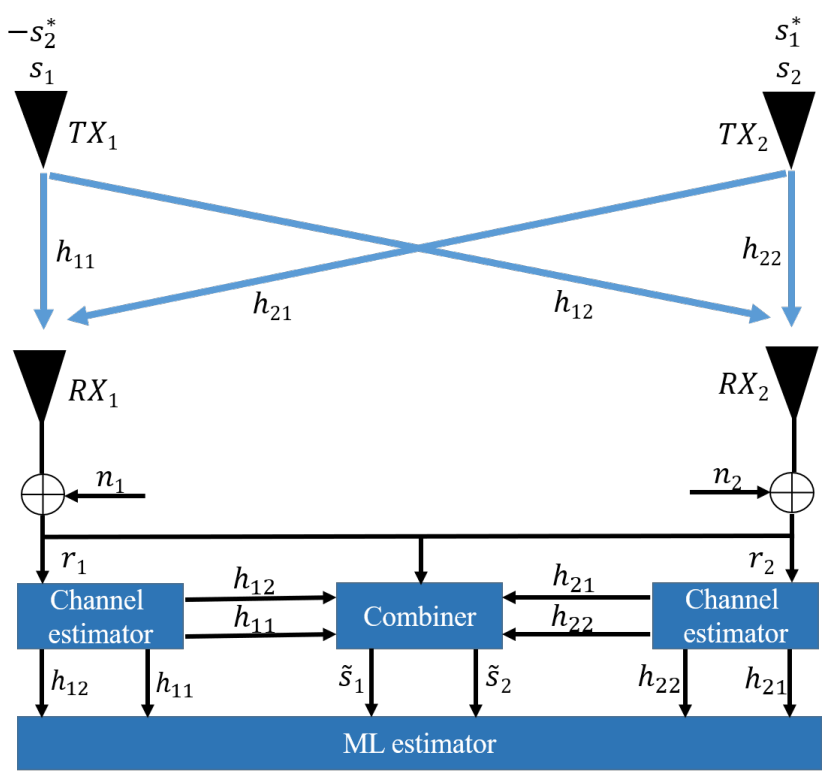

Figure 1. Illustration of $2 \times 2$ Alamouti space time coding (STC).

The Alamouti STC operation of the two symbols $s_{1}$ and $s_{2}$ is defined in Table 2 and includes two transmit antennas under two-time slots. At some time $t$, symbols $s_{1}$ and $s_{2}$ are transmitted from $T X_{1}$ and $T X_{2}$, respectively. At time $t+T_{s}$, where $T_{s}$ is the symbol duration, the symbols $-s_{2}^{*}$ and $s_{1}^{*}$ are transmitted from $T X_{1}$ and $T X_{2}$, respectively [1]. The operator $(\cdot)^{*}$ represents the complex conjugate.

Table 2. Transmission Alamouti coding operation.

\begin{tabular}{ccc}
\hline & $\boldsymbol{t}$ & $\boldsymbol{t}+\boldsymbol{T}_{\boldsymbol{s}}$ \\
\hline$T X_{1}$ & $s_{1}$ & $-s_{2}^{*}$ \\
$T X_{2}$ & $s_{2}$ & $s_{1}^{*}$ \\
\hline
\end{tabular}

The received signal $r$ and Additive White Gaussian Noise (AWGN) signal $n$ are denoted by two indexes, $r_{k}(l)$ and $n_{k}(l)$, where $k=1,2$ denotes the number of received antennas, and $l=1,2$ denotes the received signal at time $t$ or $t+T_{s}$, respectively. In Table 3 , for example, $r_{1}(1)$ denotes the received signal at time $t$ at $R X_{1}$.

Table 3. Received signal $r$ and additive white Gaussian noise (AWGN) signal $n$.

\begin{tabular}{ccc}
\hline & $\boldsymbol{t}$ & $\boldsymbol{t}+\boldsymbol{T}_{\boldsymbol{s}}$ \\
\hline \multirow{2}{*}{$R X_{1}$} & $r_{1}(1)$ & $r_{1}(2)$ \\
& $n_{1}(1)$ & $n_{1}(2)$ \\
\hline \multirow{2}{*}{$R X_{2}$} & $r_{2}(1)$ & $r_{2}(2)$ \\
& $n_{2}(1)$ & $n_{2}(2)$ \\
\hline
\end{tabular}

Figure 1 implies that the receiver equations can be described as $[1,27]$

$$
\begin{aligned}
& r_{1}(1)=h_{11} s_{1}+h_{12} s_{2}+n_{1}(1) \\
& r_{1}(2)=-h_{11} s_{2}^{*}+h_{12} s_{1}^{*}+n_{1}(2) \\
& r_{2}(1)=h_{21} s_{1}+h_{22} s_{2}+h_{2}(1) \\
& r_{2}(2)=-h_{21} s_{2}^{*}+h_{22} s_{1}^{*}+n_{2}(2)
\end{aligned}
$$


The combining rules for a $2 \times 2$ Alamouti system case are here defined as $[1,27]$

$$
\begin{aligned}
& \tilde{s}_{1}=h_{11}^{*} r_{1}(1)+h_{12} r_{1}^{*}(2)+h_{21}^{*} r_{2}(1)+h_{22} r_{2}^{*}(2) \\
& \tilde{s}_{2}=h_{12}^{*} r_{1}(1)-h_{11} r_{1}^{*}(2)+h_{22}^{*} r_{2}(1)-h_{21} r_{2}^{*}(2)
\end{aligned}
$$

Substituting (1) into (2) yields [27]

$$
\tilde{s}_{1}=\left(\sum_{i=1}^{2} \sum_{j=1}^{2}\left|h_{i j}\right|^{2}\right) s_{1}+h_{11}^{*} n_{1}(1)+h_{12} n_{1}^{*}(2)+h_{21}^{*} n_{2}(1)+h_{22} n_{2}^{*}(2)
$$

and

$$
\tilde{s}_{2}=\left(\sum_{i=1}^{2} \sum_{j=1}^{2}\left|h_{i j}\right|^{2}\right) s_{2}+h_{12}^{*} n_{1}(1)-h_{11} n_{1}^{*}(2)+h_{22}^{*} n_{2}(1)-h_{21} n_{2}^{*}(2)
$$

The estimation of each symbol $s_{1}$ and $s_{2}$ is multiplied with the norm of each complex channel gain without the other symbol's presence. Finally, the last receive step is the ML estimate. The transmitted symbol is estimated as follows [27]:

$$
\begin{aligned}
& \hat{s_{1}}=\operatorname{argmin}_{s_{1}}\left[\left|\tilde{s_{1}}-s_{1}\right|^{2}\right] \\
& \hat{s_{2}}=\operatorname{argmin}_{s_{2}}\left[\left|\tilde{s_{2}}-s_{2}\right|^{2}\right]
\end{aligned}
$$

Another way to display Equation (1) is through matrices. After mathematical operations of conjugation, the model will be $[1,27]$

$$
\vec{R}=\left[\begin{array}{l}
r_{1}(1) \\
r_{1}^{*}(2) \\
r_{2}(1) \\
r_{2}^{*}(2)
\end{array}\right]=\left[\begin{array}{cr}
h_{11} & h_{12} \\
h_{12}^{*} & -h_{11}^{*} \\
h_{21} & h_{22} \\
h_{22}^{*} & -h_{21}^{*}
\end{array}\right]\left[\begin{array}{l}
s_{1} \\
s_{2}
\end{array}\right]+\left[\begin{array}{l}
n_{1}(1) \\
n_{1}^{*}(2) \\
n_{2}(1) \\
n_{2}^{*}(2)
\end{array}\right]
$$

In short,

$$
\vec{R}=H_{T R} \vec{S}+\vec{N}
$$

where $\vec{R}$ is the receiver signal vector, $\vec{S}$ is the symbol matrix, $\vec{N}$ is the AWGN noise vector, and $H_{T R}$ is the channel matrix between the transmitter and the receiver, shown as

$$
H_{T R}=\left[\begin{array}{cc}
h_{11} & h_{12} \\
h_{12}^{*} & -h_{11}^{*} \\
h_{21} & h_{22} \\
h_{22}^{*} & -h_{21}^{*}
\end{array}\right]
$$

An important feature of this Alamouti coding matrix is the orthogonality between its columns [27]. If the condition

$$
\operatorname{column}_{1}^{H} \operatorname{column}_{2}=0
$$

is met, the vectors are orthogonal. The operator $(\cdot)^{H}$ is the complex transpose. For the $H_{T R}$ case,

$$
\left[\begin{array}{llll}
h_{11}^{*} & h_{12} & h_{21}^{*} & h_{22}
\end{array}\right] \cdot\left[\begin{array}{r}
h_{12} \\
-h_{11}^{*} \\
h_{22} \\
-h_{21}^{*}
\end{array}\right]=0 .
$$

This critical feature allows the symbols to be decoded and Equation (3) to be obtained. It can also be seen by presenting Equation (2) as the following matrix:

$$
\overrightarrow{\tilde{S}}=\left(H_{T R}\right)^{H} \vec{R}
$$


Next, (6) is substituted into the above equation to obtain

$$
\overrightarrow{\widetilde{S}}=\left(H_{T R}\right)^{H}\left(H_{T R} \vec{S}+\vec{N}\right)=\sum_{i=1}^{2} \sum_{j=1}^{2}\left|h_{i j}\right|^{2} \mathbf{I}_{2} \vec{S}+\left(H_{T R}\right)^{H} \vec{N}
$$

which is the same as Equation (3). $\mathbf{I}_{2}$ denotes an identity matrix of size 2.

In the next section, we will present the impairment of this orthogonality through interference's.

\section{Alamouti MIMO Link in the Presence of Different Interferer Techniques}

This section discusses three types of jammer strategies or interference that could be present in a certain space. Those classifications are a general interferer, a smart jammer, and a contiguous PBN jammer. We assume that the general interferer and the smart jammer have a slow-fading channel, are located in the same environment, are using OSTBC transmissions with $2 \times 2 \mathrm{MIMO}$ array, and transmits a CE with FD assume and TDD access. In addition, they have a different scheduler, i.e., a HetNet scenario [2,30].

We will discuss the interference effects on the receive symbols. Each jammer or interference technique has a different impact on the target communication system, so different Anti-Jamming (AJ) methods to cancel the jammer effects are needed. It is essential to examine the specific target's effects, especially in the mathematical design, BER, the constellation diagram, and SIR measurements. This is significant because if the same advanced cancellation technique, such as RF analog cancellation or beamforming with null-space-like spatial multiplexing [27], or interference rejection combining (IRC) algorithm [13,36] are not effective, jammer or interference symbols are received.

The issues of interference management in the Radio Access Network (RAN) and Radio Link Control (RLC), to optimize the power and spectral efficiency, are critical, especially in 5G-NR access. We have a new problem in the demodulation and the decoder process called digital interference cancellation. Comparing the different jammers is necessary because we want to model and distinguish between the different effects in BER and SIR execution.

It is essential to emphasize the effects of orthogonality violation in the spectrum, which results from the multiplicity of interferences between the transmitter and the receiver. Spacetime coding is used to achieve spatial diversity. It is possible because of the underlying propagation physics in a multi-path communication channel [27]. That is, every pair path transmits between the transmission to the receiver is sufficiently uncorrelated. In Appendix A, we analyze mathematically and illustrate the significant impairment in the orthogonal spectrum assumption.

We present a system consisting of a $2 \times 2$ Alamouti STC transmitter, TX, a receiver, $R X$, as described in the previous section, and an interferer transmitter, $J X$, as illustrated in Figure 2. We assume a far-field between the receiver and the transmitter and interference $[4,5]$.

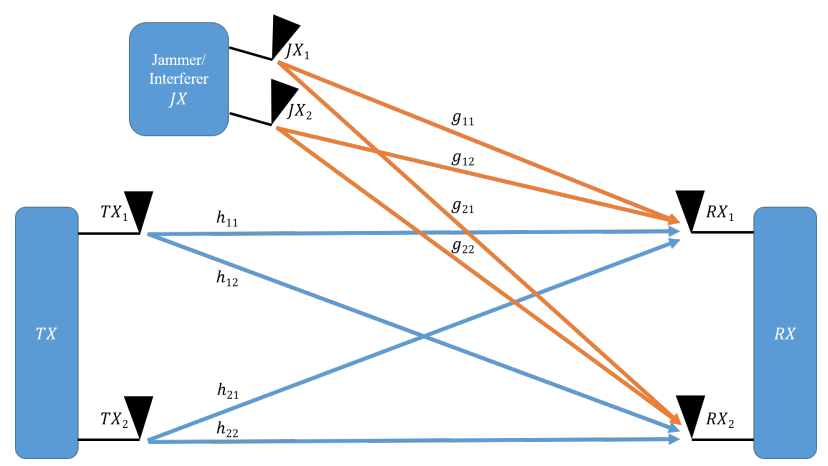

Figure 2. Illustration of $2 \times 2$ Alamouti space time coding (STC) in the presence of interference. 
In a general case where several neighboring interferences are present in the space, the received signal vector $\vec{R}$ is

$$
\vec{R}=\sqrt{P} H_{T R} \vec{S}+\sum_{l=1}^{L} \sqrt{\frac{P_{l}}{S I R_{l}}} H_{J R_{l}} \vec{J}_{l}+\sqrt{\frac{P}{S N R}} \vec{N}
$$

assuming the signal's transmission power is equal to $P$, where $L$ is the number of interferences. When considering a single dominant interference, i.e., a very high Dominant Interferer Proportion (DIP) ratio [38] the received signal vector $\vec{R}$ becomes

$$
\vec{R}=\sqrt{P} H_{T R} \vec{S}+\sqrt{\frac{P}{S I R}} H_{J R} \vec{J}+\sqrt{\frac{P}{S N R}} \vec{N}
$$

We presented expressions $H_{T R} \vec{S}$ and $\vec{N}$ in the previous section. SIR and SNR are the signal to interference and the signal to noise power ratios, respectively, at the receiver site. We will now discuss the expression $H_{J R} \vec{J}$. Like the signal, the jammer consists of two transmission antennas, $J X_{1}$ and $J X_{2}$. The jammer transmits through a MIMO channel model with four flat fading paths assumed to be independent and uncorrelated. These four paths are represented by complex channel gains $g_{11}, g_{12}, g_{21}$, and $g_{22}$. The indexes are the indexes of $J X$ and $R X$ antennas, respectively, as shown in Table 4.

Table 4. Definition of complex channel gain between the interference and receive antennas.

\begin{tabular}{ccc}
\hline & $\boldsymbol{R} \boldsymbol{X}_{\mathbf{1}}$ & $\boldsymbol{R} \boldsymbol{X}_{\mathbf{2}}$ \\
\hline$J X_{1}$ & $g_{11}$ & $g_{12}$ \\
$J X_{2}$ & $g_{21}$ & $g_{22}$ \\
\hline
\end{tabular}

The next subsection presents three representative and essential examples of interference and jammer techniques: a general interferer, a smart jammer, and a PBN jammer.

\subsection{General Interferer}

As mentioned above, the rapid rise in the need to connect thousands of wireless mobile components in a specific area is binding to improve the spectral efficiency per unit area, especially in frequency bands below $6 \mathrm{GHz}$. To achieve those high requirements, the deployment of many small cells per area is necessary $[30,31,39]$.

The mobile or modern wireless architecture has a wide range of manufacturers under license and unlicensed standards. Multiple manufacturers that communicate with UE pieces in the same local network area without sharing synchronization in the radio management or without common schedulers create serious and complicated problems. Problems include Inter-Cell Interference (ICI) [40] and Cross-Link Interference (CLI) between neighboring small cells of different directional transmissions [31]. These situations require a state of continuous decoding ability when a strong interference signal power is present, or under minimum SINR conditions [37]. A prevention situation that creates severe chain reactions such as rolling modulation and multiple handovers to far cells is also considered. False decoding of the packet detection in the front receiver process also creates a complicated situation. Even systems with a Frequency-Hoping Spread Spectrum (FHSS) scheme and a Direct Sequence Spread Spectrum (DSSS), which are embedded in protocols and standards such as Wireless Local Area Networks (WLANs), Bluetooth, and Wireless Personal Area Network (WPANs), create a situation of mutual interference and receive strong interference signals one from the other [41].

The most realistic and complicated situations are the HetNet scenario and the multiAccess Point (AP), and the multi-user scenario. These scenarios are common in 5G-NR under the dynamic TDD assumption, which produces dynamic switch point between uplinks/downlinks [42]. These scenarios create three complicated issues. The first is 
that every component, user, small cell, and macrocell base-station, can play the rule of interfering, transmit, and receiving simultaneously. The second involves the complexity of un-decode-ability in the presence of strong interference signals [37], much more so than the SIR threshold to achieve a discovery production threshold. Third, distinguishing between data symbols and interference symbols when combining multi-interference signals with different power levels that are out of synchronization with discovery times [32], or hidden beyond a powerful interference signal, is also very challenging.

This subsection describes a general interferer's specific case based on these scenarios, combined with the Alamouti-STC scheme. The mathematics analysis in the frequency domain contributes to a critical study that describes the impact of synchronization errors on Alamouti-STC-based cooperative MIMO schemes [32]. In this analysis, we describe UE transmitting an Alamouti-STC scheme, with the same assumptions made at the beginning of this section, to other UEs that act as a general interferer or a general jammer device. That jammer or interferer also transmits the Alamouti-STC. The interferer symbols $j_{1}$ and $j_{2}$ are defined in Table 5.

Table 5. Transmission Alamouti coding operation for the jammer.

\begin{tabular}{ccc}
\hline & $t$ & $t+T_{s}$ \\
\hline$J X_{1}$ & $j_{1}$ & $-j_{2}^{*}$ \\
$J X_{2}$ & $j_{2}$ & $j_{1}^{*}$ \\
\hline
\end{tabular}

We therefore can write Equation (10) as

$$
\vec{R}=\left[\begin{array}{l}
r_{1}(1) \\
r_{1}^{*}(2) \\
r_{2}(1) \\
r_{2}^{*}(2)
\end{array}\right]=\sqrt{P}\left[\begin{array}{cc}
h_{11} & h_{12} \\
h_{12}^{*} & -h_{11}^{*} \\
h_{21} & h_{22} \\
h_{22}^{*} & -h_{21}^{*}
\end{array}\right]\left[\begin{array}{l}
s_{1} \\
s_{2}
\end{array}\right]+\sqrt{\frac{P}{S I R}}\left[\begin{array}{cc}
g_{11} & g_{12} \\
g_{12}^{*} & -g_{11}^{*} \\
g_{21} & g_{22} \\
g_{22}^{*} & -g_{21}^{*}
\end{array}\right]\left[\begin{array}{l}
j_{1} \\
j_{2}
\end{array}\right]+\sqrt{\frac{P}{S N R}}\left[\begin{array}{l}
n_{1}(1) \\
n_{1}^{*}(2) \\
n_{2}(1) \\
n_{2}^{*}(2)
\end{array}\right]
$$

The receiver equations are

$$
\begin{aligned}
& r_{1}(1)=h_{11} s_{1}+h_{12} s_{2}+g_{11} j_{1}+g_{12} j_{2}+n_{1}(1) \\
& r_{1}^{*}(2)=h_{12}^{*} s_{1}-h_{11}^{*} s_{2}+g_{12}^{*} j_{1}-g_{11}^{*} j_{2}+n_{1}^{*}(2) \\
& r_{2}(1)=h_{21} s_{1}+h_{22} s_{2}+g_{21} j_{1}+g_{22} j_{2}+n_{2}(1) \\
& r_{2}^{*}(2)=h_{22}^{*} s_{1}-h_{21}^{*} s_{2}+g_{22}^{*} j_{1}-g_{21}^{*} j_{2}+n_{2}^{*}(2)
\end{aligned}
$$

Substituting (12) into the combining Equation (2) yields

$$
\begin{aligned}
\tilde{s}_{1}= & \left(\sum_{i=1}^{2} \sum_{j=1}^{2}\left|h_{i j}\right|^{2}\right) s_{1}+h_{11}^{*} n_{1}(1)+h_{12} n_{1}^{*}(2)+h_{21}^{*} n_{2}(1)+h_{22} n_{2}^{*}(2)+ \\
& +j_{1}\left(h_{11}^{*} g_{11}+h_{12} g_{12}^{*}+h_{21}^{*} g_{21}+h_{22} g_{22}^{*}\right)+j_{2}\left(h_{11}^{*} g_{12}-h_{12} g_{11}^{*}+h_{21}^{*} g_{22}-h_{22} g_{21}^{*}\right) \\
& \text { and } \\
\tilde{s}_{2}= & \left(\sum_{i=1}^{2} \sum_{j=1}^{2}\left|h_{i j}\right|^{2}\right) s_{2}+h_{12}^{*} n_{1}(1)-h_{11} n_{1}^{*}(2)+h_{22}^{*} n_{2}(1)-h_{21} n_{2}^{*}(2)+ \\
& +j_{1}\left(h_{12}^{*} g_{11}-h_{11} g_{12}^{*}+h_{22}^{*} g_{21}-h_{21} g_{22}^{*}\right)+j_{2}\left(h_{12}^{*} g_{12}+h_{11} g_{11}^{*}+h_{22}^{*} g_{22}+h_{21} g_{21}^{*}\right)
\end{aligned}
$$

It can be seen that, compared to Equation (3), we received, in addition to the symbols, expressions containing combinations of the interferer with the channel components. As a result, errors in detecting the symbols and a decrease in performance will be obtained. In Section 4, we will present simulation results for representative cases of this phenomenon. 


\subsection{Smart Jammer}

When we describe the smart jammer principle, it is critical to know the RAN behavior and the protocols that manage the PHY layer of the target wireless communication system's air interface. A smart jammer has two main guiding principles. The first one is the ability to estimate the channel matrix or the CSI between the UE to the base-station (e/gNodeB), and between the jammer to this base-station (NodeB), from the jammer's perspective [21]. An example is a smart jammer technique called a pilot-nulling attack. The adaptive process is called Battle Damage Assessment (BDA) [43]. Another example is a jammer that joins a network to legitimize the UE and manipulate the RF PHY layer. This is very destructive and effective against the communication system. The second principle is communication deterioration by converting the target system's advantage to the most significant disadvantage [27]. An excellent example of this scenario is the exploitation of the channel's multiplicity of paths that characterizes Rayleigh fading. This exploitation is manifested in the fact that, with advanced MIMO techniques, we cause in the receiver processing a situation of linear independence between every path [27]. If the smart jammer hits this principle, many interference signals will be involved in the de-modulator and the combiner at the receive side. It leads to a destructive chain reaction that begins with a decrease in the modulation order that extends the transmission time and causes a decrease in BER performance.

In this subsection, we describe the communication model of combining a smart jammer using the Alamouti-STC MIMO model, which is a $2 \times 2$ MIMO array. Based on the assumption that the smart jammer is estimating the channel matrix, $H_{T R}$, between the UE to the base-station (NodeB), we can write Equation (11) as

$$
\vec{R}=\left[\begin{array}{l}
r_{1}(1) \\
r_{1}^{*}(2) \\
r_{2}(1) \\
r_{2}^{*}(2)
\end{array}\right]=\sqrt{P}\left[\begin{array}{rr}
h_{11} & h_{12} \\
h_{12}^{*} & -h_{11}^{*} \\
h_{21} & h_{22} \\
h_{22}^{*} & -h_{21}^{*}
\end{array}\right]\left[\begin{array}{l}
s_{1} \\
s_{2}
\end{array}\right]+\sqrt{\frac{P}{S I R}}\left[\begin{array}{rr}
h_{11} & h_{12} \\
h_{12}^{*} & -h_{11}^{*} \\
h_{21} & h_{22} \\
h_{22}^{*} & -h_{21}^{*}
\end{array}\right]\left[\begin{array}{l}
j_{1} \\
j_{2}
\end{array}\right]+\sqrt{\frac{P}{S N R}}\left[\begin{array}{l}
n_{1}(1) \\
n_{1}^{*}(2) \\
n_{2}(1) \\
n_{2}^{*}(2)
\end{array}\right]
$$

After normalizing the powers, the receive equations are

$$
\begin{aligned}
& r_{1}(1)=h_{11}\left(s_{1}+j_{2}\right)+h_{12}\left(s_{2}+j_{1}\right)+n_{1}(1) \\
& r_{1}^{*}(2)=h_{12}^{*}\left(s_{1}-j_{2}\right)-h_{11}^{*}\left(s_{2}-j_{1}\right)+n_{1}^{*}(2) \\
& r_{2}(1)=h_{21}\left(s_{1}+j_{2}\right)+h_{22}\left(s_{2}+j_{1}\right)+n_{2}(1) \\
& r_{2}^{*}(2)=h_{22}^{*}\left(s_{1}-j_{2}\right)-h_{21}^{*}\left(s_{2}-j_{1}\right)+n_{2}^{*}(2)
\end{aligned}
$$

If we are substituting (15) into the combiner rule at the receiver (2), it yields

$$
\begin{aligned}
\tilde{s}_{1}= & \left(\sum_{i=1}^{2} \sum_{j=1}^{2}\left|h_{i j}\right|^{2}\right) s_{1}+h_{11}^{*} n_{1}(1)+h_{12} n_{1}^{*}(2)+h_{21}^{*} n_{2}(1)+h_{22} n_{2}^{*}(2)+ \\
& +j_{2}\left(\left|h_{11}\right|^{2}-\left|h_{12}\right|^{2}+\left|h_{21}\right|^{2}-\left|h_{22}\right|^{2}\right)
\end{aligned}
$$

and

$$
\begin{aligned}
\tilde{s}_{2}= & \left(\sum_{i=1}^{2} \sum_{j=1}^{2}\left|h_{i j}\right|^{2}\right) s_{2}+h_{12}^{*} n_{1}(1)-h_{11} n_{1}^{*}(2)+h_{22}^{*} n_{2}(1)-h_{21} n_{2}^{*}(2)+ \\
& +j_{1}\left(-\left|h_{11}\right|^{2}+\left|h_{12}\right|^{2}-\left|h_{21}\right|^{2}+\left|h_{22}\right|^{2}\right)
\end{aligned}
$$

The result obtained is impressive because the interference, multiplied by the norms, was obtained in addition to the symbol, i.e., the interfering symbol was added. In Section 4, we will present simulation results for representative cases of this phenomenon. 


\subsection{Partial Band Noise Jammer}

The PBN jammer [44] places noise jammer energy across a specific portion of the entire system's target bandwidth. For example, Figure 3 shows a 50\% PBN jammer, i.e., $50 \%$ of the target bandwidth noise is added. PBN is a straightforward jammer technique in the modern communication world. PBN does not require any computational estimation or channel estimation between the target from the jammer's perspective and the receiver or any knowledge about the time synchronization of the target's preamble packet.

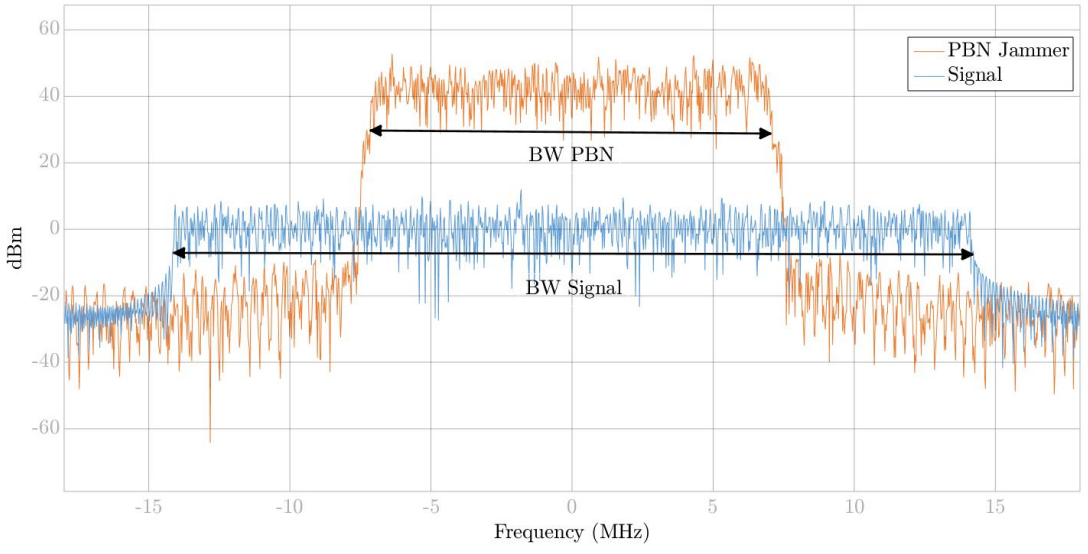

Figure 3. Demonstration of the $50 \%$ bandwidth (BW) partial band noise (PBN) jammer.

The influence of the PBN jammer on the receiver in the space domain can be negligible if we use MIMO techniques such as Spatial Multiplexing (SM) decoding technique's, Minimum Variance Distortion-less Response (MVDR), and dynamic beamforming, especially if the channel state information is known to the UE side [27]. The selectivity of the PBN in the space domain depends on the PBN variance and bandwidth and on the signal noise power. In order to reduce the effect of the PBN jammer, advanced MIMO techniques at the space domain can be used $[27,28]$.

\section{Numerical Results}

We performed simulations of the $2 \times 2$ Alamouti STC in the time-space domain with the presence of a general interferer, a smart jammer, and a PBN jammer, described in Sections 3.1, 3.2 and 3.3, respectively. The simulations were performed in both Matlab and Simulink platforms.

The simulation general block diagram is described in Figure 4, and the Simulink block diagram is illustrated in Figure 5. In this experiment, we focused on two leading parameters that reflect the system performance: BER and SIR. The transmission chains have a data block generator. Next, the data enter a modulator block. We chose the MPSK modulation, but other modulations can be selected. The M-PSK signals have equal energy, i.e., a Constant Envelope (CE), which is energetically efficient, and propose low PAPR pre-coding [45]. The STC encoder encodes the symbol sequence to obtain $\vec{S}$ and $\vec{J}$ and transmits on two antennas. The MIMO block filters the signals through an MIMO multi-path Rayleigh slow-fading channel. At the receiver, two antennas receive the signal vector $\vec{R}$ where the reverse operations are performed: the STC combiner and the M-PSK demodulator. As we mentioned in the introduction, we assume a private case of the HetNet scenario, which included TDD network accesses and an FD mode at the receiver, the transmitter, and with un-shared scheduler assume. The presence of the interference signals is continual. 


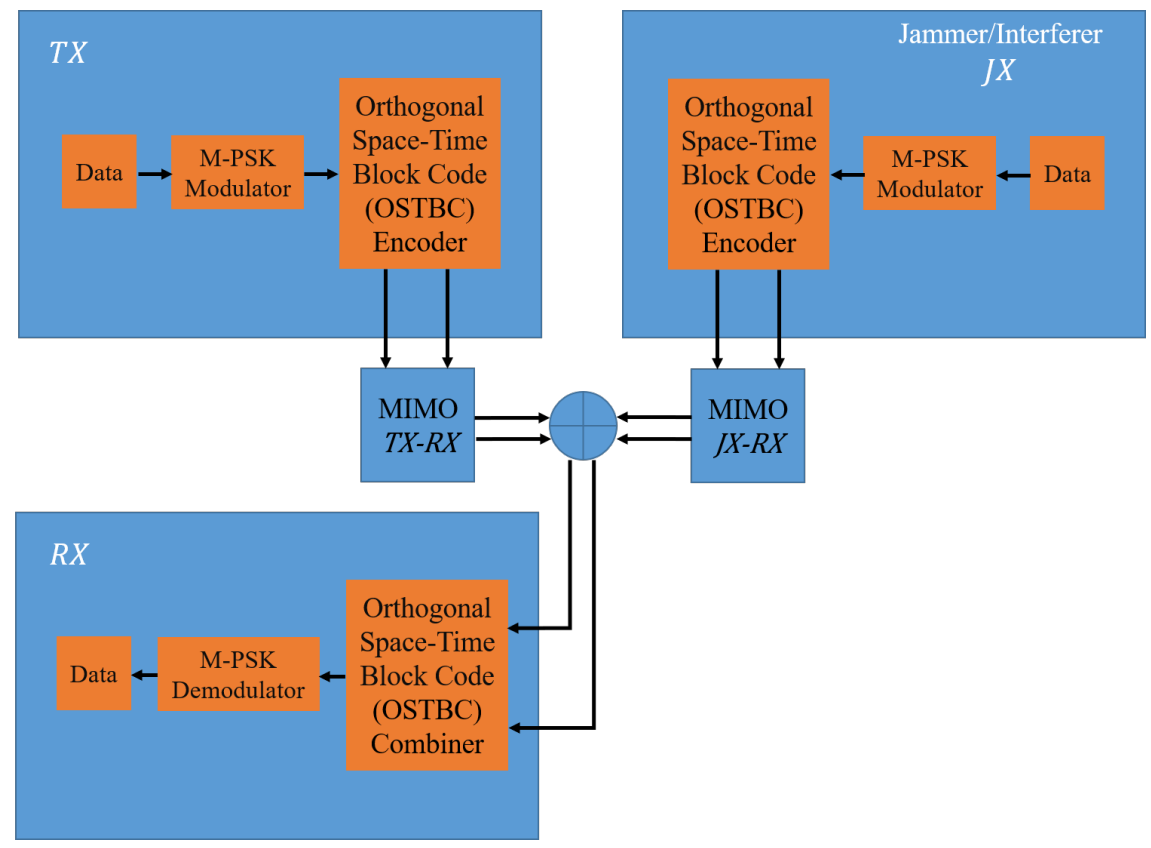

Figure 4. Simulation general block diagram of the signal and interferer using a $2 \times 2$ Alamouti technique.

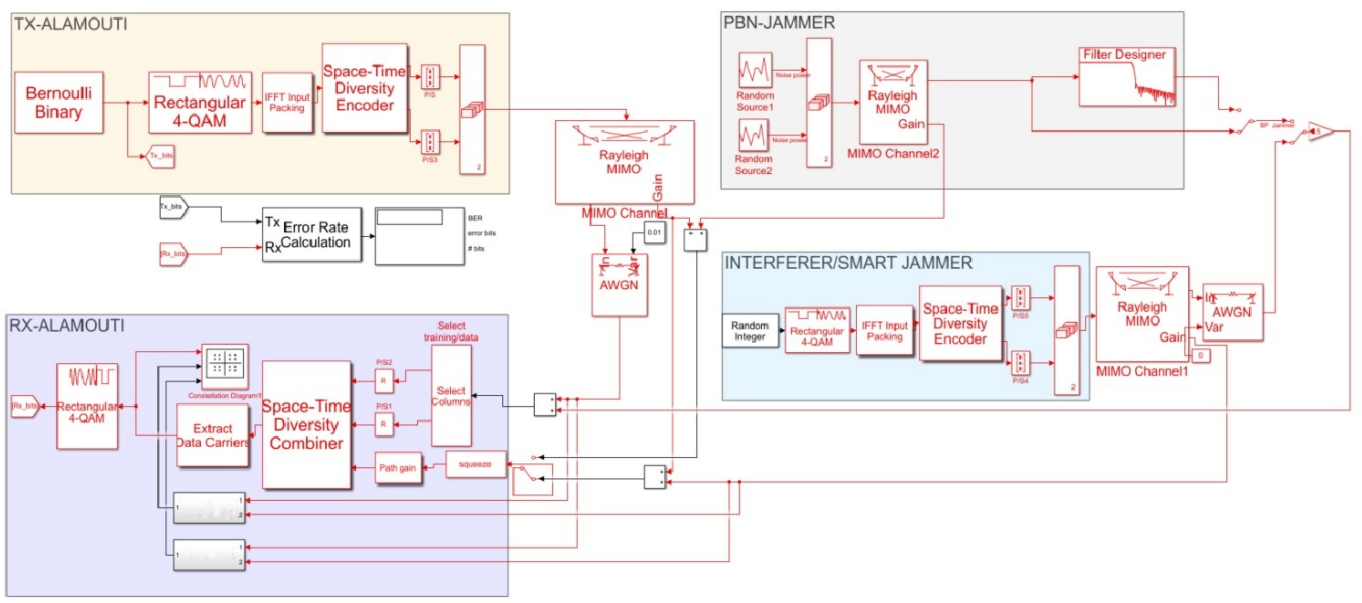

Figure 5. Simulation block diagram using the Simulink platform.

\subsection{General Interferer}

We first simulated the case described in Section 3.1. This is the general case. The received symbols are presented in (13). Figure 6 shows the constellation of the transmitted symbols in blue and the received symbols in orange when sending 1000 bits with 4, 8 , 16-PSK modulations with no AWGN and SIR $=10 \mathrm{~dB}$. The received symbols are random and depend on the channel matrix's characteristics, $H_{T R}$ and $H_{J R}$. These values are random and independent. Therefore, we obtain random receives symbol results, i.e., the general interferer creates random byproducts in the combining block. This issue poses a difficulty in constructing cost terms for the discovery of the symbols. 


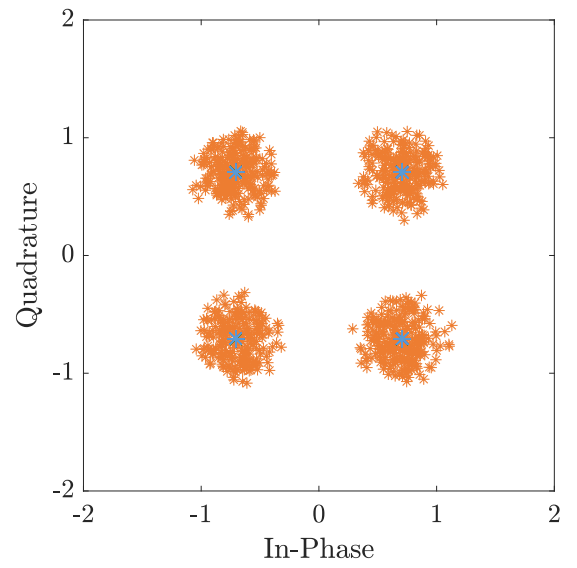

(a)

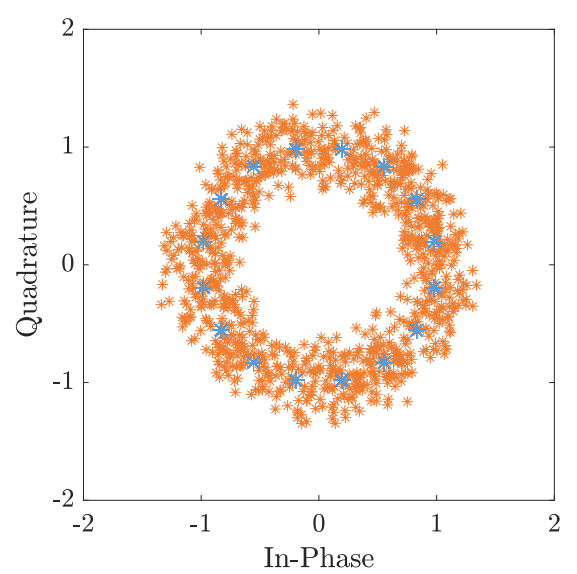

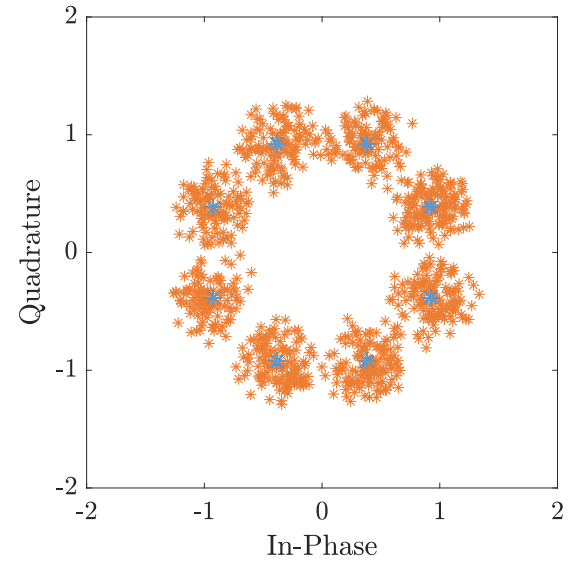

(b)

(c)

Figure 6. A constellation of transmitted symbols (blue) and received symbols in the presence of the general interferer (orange), for (a) 4-Phase-Shift Keying (PSK), (b) 8-PSK, (c) 16-PSK modulations, no Additive White Gaussian Noise (AWGN), and SIR = $10 \mathrm{~dB}$.

An interesting innovation to examine is the general interferer effect on system performance in terms of Bit Error Rate (BER). The BER performances as a function of SIR are shown in Figure 7 . For $M=2,4,8,16-P S K$ constellation orders, SNR $=15 \mathrm{~dB}$, with $10^{5}$ transmitted bits, and $\Delta \mathrm{SIR}=0.2 \mathrm{~dB}$.

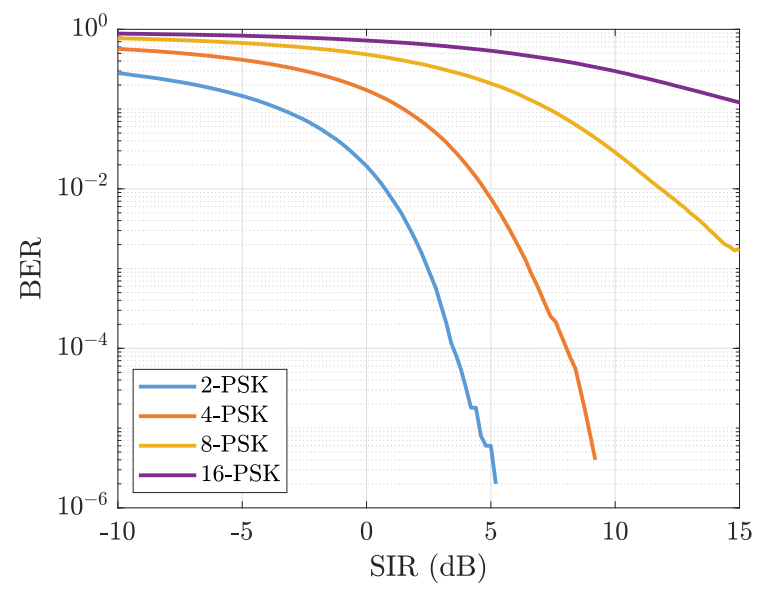

Figure 7. Alamouti $2 \times 2$ with the presence of the general interferer. Bit error rate (BER) as a function of the signal to interference power ratio (SIR), for different modulation orders 2, 4, 8, 16-PSK and $\mathrm{SNR}=15 \mathrm{~dB}$. 
As expected, a higher modulation order leads to a lower BER performance because the distance between the transmitted symbols is shorter, as shown in Figure 6. In addition, a higher modulation order increases DG, i.e., the shift of the graphs with respect to a reference curve with $\mathrm{DO}$ of 2 [27].

\subsection{Smart Jammer}

Next, we simulated the case described in Section 3.2. The received symbols are presented in (16). Figure 8 shows the transmitted and received symbols for the 4, 8, 16-PSK modulations without AWGN, sending 1000 bits, and SIR = $10 \mathrm{~dB}$. It can be seen that the received symbols are replicated according to the modulation order of the interferer, i.e., for a 4-PSK modulation, around each symbol transmitted, four symbols will be received in a 4-PSK constellation. For an 8-PSK modulation, around each symbol eight symbols will be received in an 8-PSK constellation, and, in the same way, will be accepted for other modulations. This phenomenon corresponds to (16) in which, in addition to the transmitted signal, the interference is obtained multiplied by a real number.

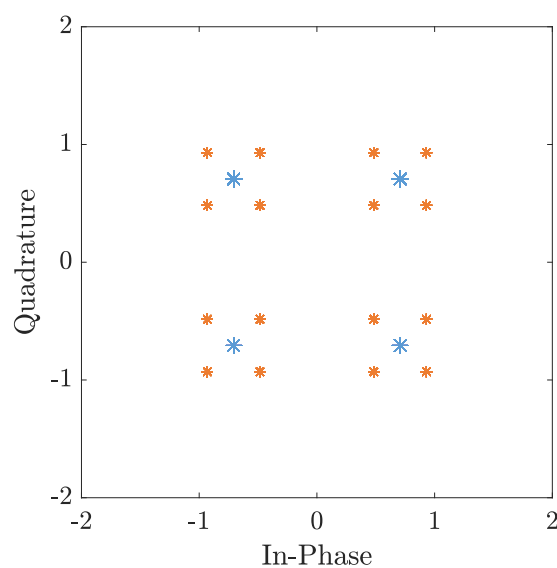

(a)

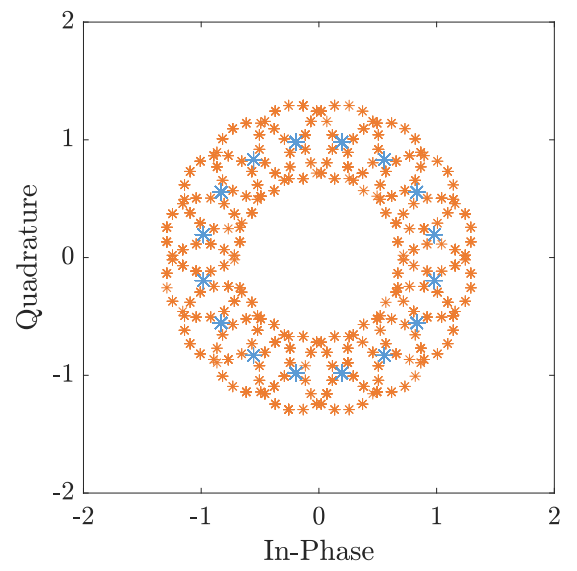

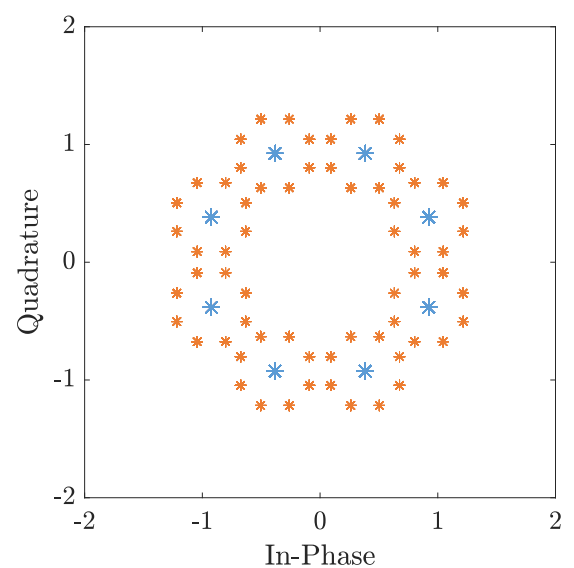

(b)

(c)

Figure 8. A constellation of transmitted symbols (blue) and received symbols in the presence of the smart jammer (orange), for (a) 4-PSK, (b) 8-PSK, (c) 16-PSK modulations, no AWGN, and $\mathrm{SIR}=10 \mathrm{~dB}$.

The BER performances as a function of SIR are shown in Figure 9. For $M=2,4,8$, 16-PSK constellation orders, SNR $=15 \mathrm{~dB}$, with $10^{5}$ transmitted bits, and $\triangle \mathrm{SIR}=0.2 \mathrm{~dB}$. 


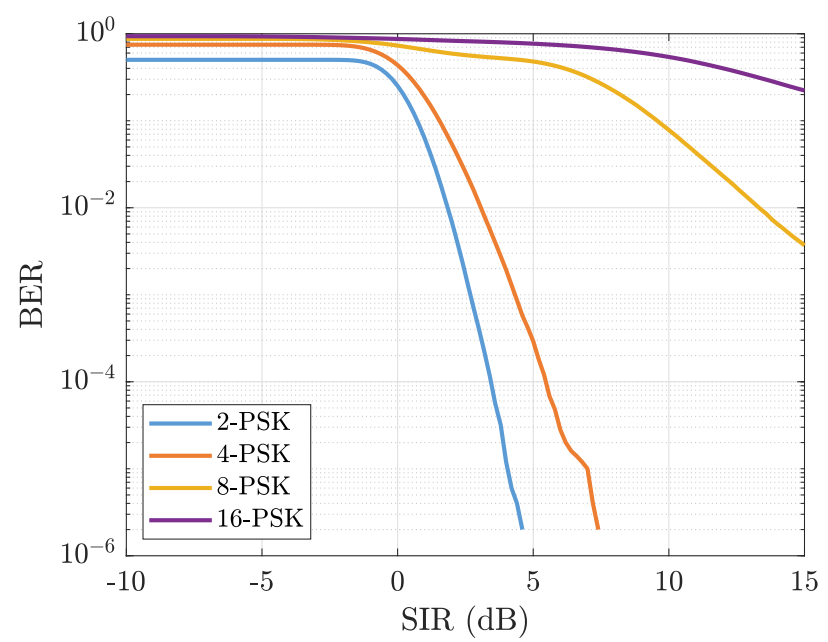

Figure 9. Alamouti $2 \times 2$ with the presence of the smart jammer. BER as a function of the SIR. Different modulation orders 2, 4, 8, 16-PSK and SNR $=15 \mathrm{~dB}$.

For low SIR values, in the graphs of 4, 8, 16-PSK modulations, a BER greater than 0.5 is obtained. This can be explained by Figure $8 \mathrm{c}$. In this graph, it can be seen that the received symbols are next to the neighboring symbols and, therefore, will inevitably be incorrectly demodulated. As a result, the probability of the resulting error is greater than 0.5. This phenomenon does not occur in 2-PSK modulation because, in this modulation, the symbols have no neighbors on either side but only on one side. In addition, for low SIR values, the graphs are horizontal. This phenomenon also occurs because of symbol duplication. The SIR value affects the distance between the transmitted symbols and those received. When SIRs are low, the received symbols are more distant and therefore located in their neighbor's symbols; however, there is a particular value of SIR in which they will no longer slide to their neighbors. As a result, the BER performance will improve sharply and noticeably, as indicated in Figure 9.

\subsection{Partial Band Noise Jammer}

Next, we simulated the case described in Section 3.3. For this, we used a 50\% PBN jammer. Figure 10 shows the constellation of the transmitted symbols in blue and the received symbols in orange when sending 1000 bits with 4, 8, 16-PSK modulations with no $\mathrm{AWGN}$ and SIR $=10 \mathrm{~dB}$. As expected, the received symbols are scattered randomly around the transmitted symbols as a result of adding PBN. The scattering distance from the transmitted symbols depends on the SIR. The lower its value is, the wider the scatter will be, and vice versa.

The results are similar to the case of the general interferer. The difference between the general case and the PBN case is in the ML estimator processing or the SD block. If we have a symbol $s_{2}$ that satisfies the necessary condition search, we construct a sufficient condition for symbol $s_{1}$. In the general case, we have a greater minimization problem from the PBN case because symbol $s_{2}$ affects whether symbol $s_{1}$ can be found. If there is an impairment in the orthogonality between the channel-matrix columns, as described in the general interferer case, and also in the Appendix A, then the symbol $s_{2}$ will depend on finding the symbol $s_{1}$. In ML and SD, the search terms are defined by a circle equation. When there is a violation of the orthogonality, a circle in the $s_{1}$ plane is built with a center and radius that depend on $s_{2}$. 


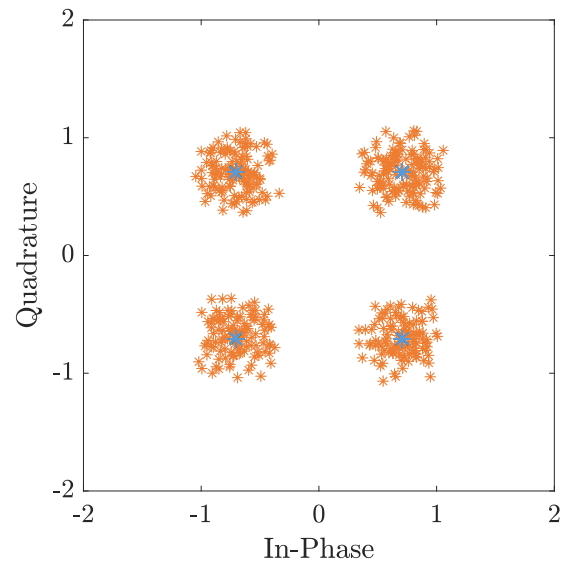

(a)

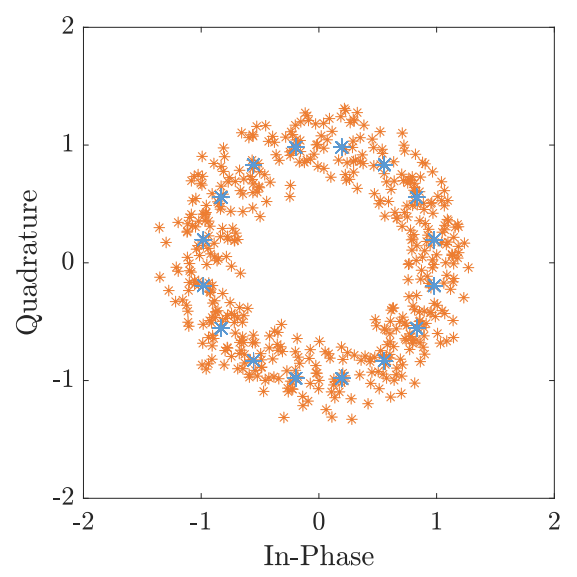

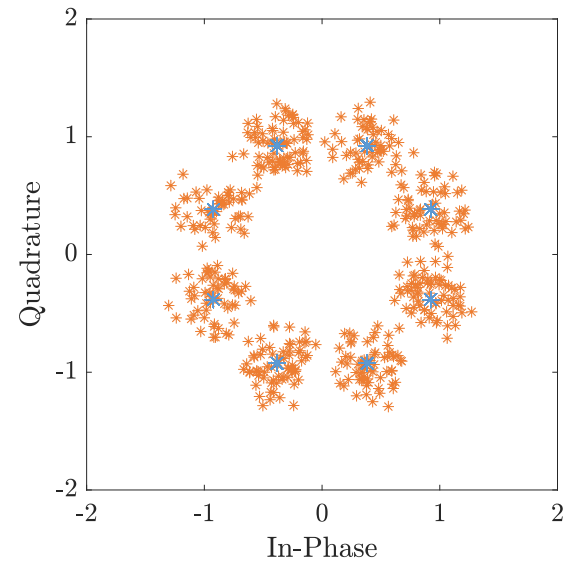

(b)

(c)

Figure 10. A constellation of transmitted symbols (blue) and received symbols in the presence of the partial band noise (PBN) jammer (orange), for (a) 4-PSK, (b) 8-PSK, (c) 16-PSK modulations, no $\mathrm{AWGN}$, and SIR $=10 \mathrm{~dB}$.

Figure 11 shows the BER graphs as a function of the SIR when SNR $=15 \mathrm{~dB}$. Several PSK constellation orders, $\mathrm{M}=2,4,8,16$, were considered, $10^{5}$ bits were transmitted, and $\Delta \mathrm{SIR}=0.2 \mathrm{~dB}$.

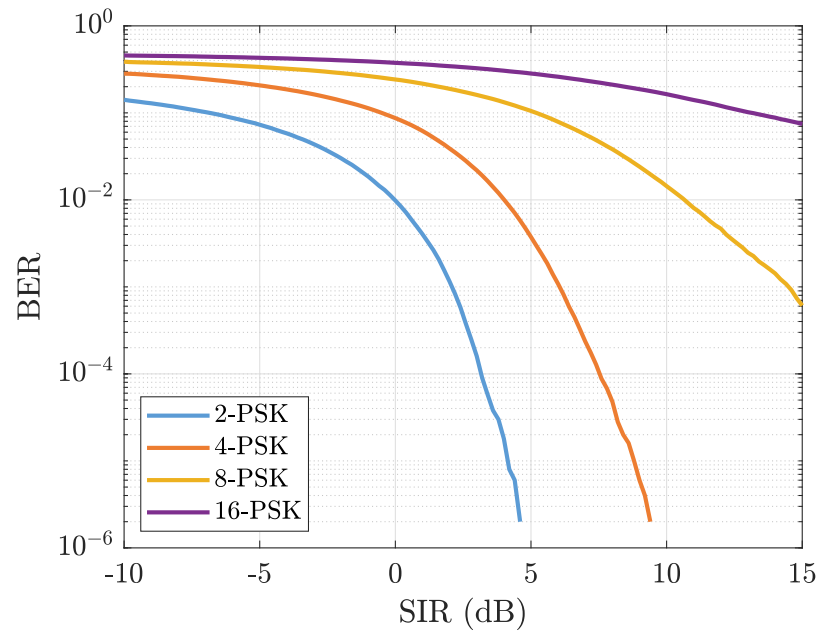

Figure 11. Alamouti $2 \times 2$ with the presence of the partial band noise (PBN) jammer. BER as a function of the SIR. Different modulation orders 2, 4, 8, 16-PSK and SNR $=15 \mathrm{~dB}$. 
As in the case of the general interferer, the results show that a higher modulation order leads to lower BER.

Finally, and most importantly, in Figure 12, we compared the different types of interferers concerning the 4-PSK modulation case. For SIR values smaller than $2 \mathrm{~dB}$, the most destructive is the smart jammer due to duplicate symbols, followed by a general interferer and finally the PBN jammer. As mentioned, the PBN we used is of the $50 \%$ type, so its BER outperforms that of the general jammer. For SIR values greater than $2 \mathrm{~dB}$, the smart jammer's performance is the most reliable, and the performance of the general interferer and the PBN jammer becomes similar. As we have seen, the smart jammer graphs are horizontal when the SIR values are negative. In this range, even if we increase the SIR value by, for example, a null space direct to the smart jammer to achieve selectivity in the space domain, performance will not improve, unlike the other interferer.

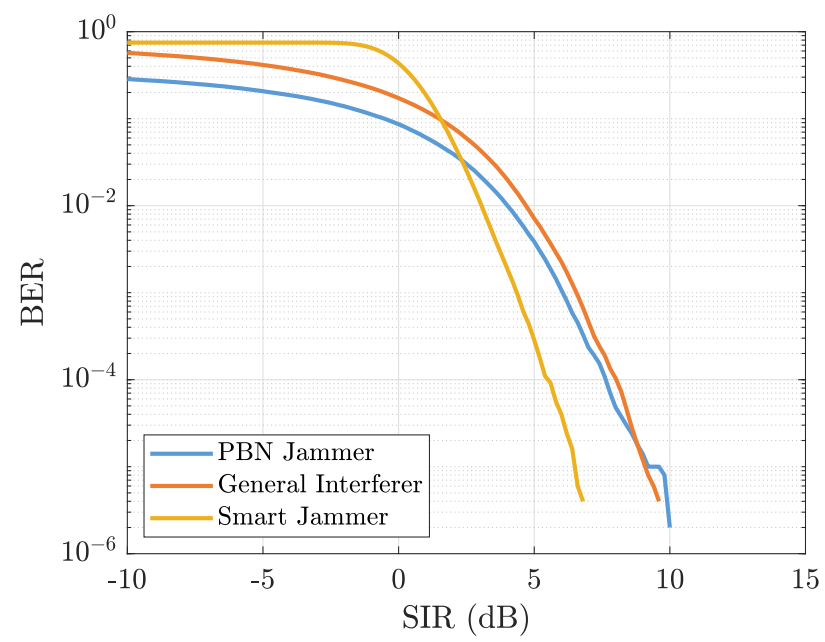

Figure 12. Comparison of BER as a function of the SIR for different types of interferers, 4-PSK modulation, and SNR $=15 \mathrm{~dB}$.

Simulation parameters of all the above figures of the BER performances as a SIR function are summarized in Table 6.

Table 6. Simulations parameters.

\begin{tabular}{cc}
\hline Parameter & Value/Description \\
\hline Transmission antennas & 2 \\
Receiving antennas & 2 \\
Space-time code & Alamouti \\
Duplex mode & TDD \\
Fading model & Rayleigh \\
transmitted bits & $10^{5}$ \\
Modulation & $2,4,8,16-\mathrm{PSK}$ \\
SNR & $15 \mathrm{~dB}$ \\
SIR & $-10 \mathrm{~dB}$ to $15 \mathrm{~dB}$ \\
$\Delta$ SIR & $0.2 \mathrm{~dB}$ \\
\hline
\end{tabular}

From the BER results depending on the SIR obtained in this chapter, the Channel Quality Indicator (CQI) value can be estimated for the different cases [46]. This value, at 5G-NR, keeps the BER below a pre-defined threshold for each user by determines the order and type of modulations, the code rate and the spectral efficiency of the base station downlink transmission. 


\section{Conclusions}

In this paper, we study and present the significant impact and performances of the Alamouti scheme in the presence of interferences signals. We assume that the channel is under Rayleigh slow fading, with CE modulation transmission. Understanding the effects of interference in the space-frequency domain is critical for developing a solution to cancel the interference by digital, analog, or antenna separation.

We proved mathematically (Appendix A), and with a simulation platform, the MIMOsystem based on the STC scheme performances in an interference environment decreases. When the CSI is only on the receiver side, it has destructive effects on the receiving and the decoding processes. We showed the violation of the principle of orthogonality that OSTBC produces, by creating uncorrelated channel paths and receiving every path with each antenna, independently. This is a significant issue in a multi-path scenario or urban environment because if all paths received are orthogonal to each other, a full rank in the channel matrix is obtained. Full rank leads to a maximum capacity in the channel, achieved by the sum of constant eigenvalues. The examination of the general interference, the smart jammer, and the PBN jammer showed an impact on the orthogonality region and caused the addition and duplication of received symbols. These phenomena are independent of the DOA, i.e., the transmitter, receiver, and interferer orientation, under a ULA assume.

We showed the BER performances in relation to the SIR in the presence of interference. We showed that it is unable to reduce the BER performances for the smart jammer case unless the SIR value is above $0 \mathrm{~dB}$. To overcome this, we must ensure that the CSI will be on the transmission side, and we need to make sure that the CSI will be on both sides at the same time.

We chose to focus on the simple case with a single dominant interferer because already, in this case, we prove mathematically and with simulations performances impairments that justify identification, control and feedback. When dealing with complex problems such as multi-interference, as occurs in reality, it is necessary to simplify the problem, build models, and then deal with the complex problems.

Our subsequent studies will aim to reach a breakthrough that enables the creation of fast, smart, and learning feedback. This feedback will be performed using an algorithm that learns the channels' characteristics between the transmitter and the interference's to the receiver, very fast and computes smart feedback between the receiver to the transmitter. This feedback must consider the trade-off between the ability to reduce and the ability to optimize energy-efficient power transmissions in the transmitter, and thus solve the classic full rank optimization problem of the interference cancellation in the receiver.

We must research and develop models and algorithms that can design dynamicselective channels while executing control processes, decoding SM techniques, cancellation multi-interference's signals, and can produce corrections in a unique geometric array of antennas. This solution is needed in a system with TDD techniques and an FD system, especially in HetNets sub $6 \mathrm{GHz}$, such as 5G-NR small cells under a multi-interference environment or military systems that suffer from smart jammers. In the future, we want to fix these problems, in addition to selective-fast-fading channels, and multi-interference with high-order modulation.

Author Contributions: Conceptualization, A.E.; methodology, A.E.; software, A.E. and Y.B.; validation, A.E.; formal analysis, A.E. and Y.B.; investigation, A.E. and Y.B.; resources, A.E.; data curation, A.E. and Y.B.; writing — original draft preparation, A.E. and Y.B.; writing-review and editing, A.E., Y.B., and Y.P.; visualization, Y.B.; supervision, Y.P.; project administration, Y.P.; All authors have read and agreed to the published version of the manuscript.

Funding: This research received no external funding.

Conflicts of Interest: The authors declare no conflict of interest. 


\section{Appendix A}

This appendix discusses the mathematical analysis of the interfering effect on the channel matrix orthogonality. Assuming all transmissions signals and interferers are $\mathrm{M}-$ PSK modulated, which have equal energy, i.e., CE, we can replace any symbol $j_{1}, j_{2}$ with any other symbol, such as $s_{1}$ and $s_{2}$, by multiplying it by $e^{j \theta}$, for $\theta \in\left\{e^{j \frac{\pi}{M}(1+2 n)}\right\}$.

We can write

$$
\left[\begin{array}{l}
j_{1} \\
j_{2}
\end{array}\right]=\left[\begin{array}{cc}
e^{j \theta_{1}} & 0 \\
0 & e^{j \theta_{2}}
\end{array}\right]\left[\begin{array}{l}
s_{1} \\
s_{2}
\end{array}\right] .
$$

We therefore can write Equation (11) as

$$
\vec{R}=\left[\begin{array}{l}
r_{1}(1) \\
r_{1}^{*}(2) \\
r_{2}(1) \\
r_{2}^{*}(2)
\end{array}\right]=\sqrt{P}\left(\left[\begin{array}{cc}
h_{11} & h_{12} \\
h_{12}^{*} & -h_{11}^{*} \\
h_{21} & h_{22} \\
h_{22}^{*} & -h_{21}^{*}
\end{array}\right]+\sqrt{\frac{1}{S I R}}\left[\begin{array}{rr}
g_{11} & g_{12} \\
g_{12}^{*} & -g_{11}^{*} \\
g_{21} & g_{22} \\
g_{22}^{*} & -g_{21}^{*}
\end{array}\right]\left[\begin{array}{cc}
e^{j \theta_{1}} & 0 \\
0 & e^{j \theta_{2}}
\end{array}\right]\right)\left[\begin{array}{l}
s_{1} \\
s_{2}
\end{array}\right]+\sqrt{\frac{P}{S N R}}\left[\begin{array}{l}
n_{1}(1) \\
n_{1}^{*}(2) \\
n_{2}(1) \\
n_{2}^{*}(2)
\end{array}\right]
$$

The combined response from the transmitter and jammer, as observed from the $R X$, can be considered the sum of two matrices acting on the same vector of symbols $\vec{S}$. Equation (A1) presents the channel matrix that includes signal transmission and interference. For simplicity, let

$$
\left[\begin{array}{ll}
v_{1} & v_{2}
\end{array}\right]=\left[\begin{array}{rr}
h_{11} & h_{12} \\
h_{12}^{*} & -h_{11}^{*} \\
h_{21} & h_{22} \\
h_{22}^{*} & -h_{21}^{*}
\end{array}\right]
$$

denote the channel matrix of the UE, and let

$$
\left[\begin{array}{ll}
w_{1} & w_{2}
\end{array}\right]=\frac{1}{\sqrt{S I R}}\left[\begin{array}{rr}
g_{11} & g_{12} \\
g_{12}^{*} & -g_{11}^{*} \\
g_{21} & g_{22} \\
g_{22}^{*} & -g_{21}^{*}
\end{array}\right]
$$

denote the channel matrix of the interference. The NodeB, $R X$, receives a signal that is the sum of the UE transmission TX and the interference transmission JX. The resulting channel matrix, after filtering the AWGN, is therefore

$$
\left[\begin{array}{ll}
v_{1} & v_{2}
\end{array}\right]+\left[\begin{array}{ll}
w_{1} & w_{2}
\end{array}\right]\left[\begin{array}{cc}
e^{j \theta_{1}} & 0 \\
0 & e^{j \theta_{2}}
\end{array}\right]
$$

which we assume to be $d \times 2$, where $v_{2}^{*} v_{1}=0$ and $w_{2}^{*} w_{1}=0$.

Recall that for two vectors $u, v \in \mathbb{C}, u, v \neq 0$, we have $\frac{\left|u^{*} v\right|}{\|u\| \cdot\|v\|} \leq 1$. Therefore, we can write $\frac{\left|u^{*} v\right|}{\|u\| \cdot\|v\|}=\cos (\varphi)$, for some $\varphi \in\left[-\frac{\pi}{2}, \frac{\pi}{2}\right]$.

The columns of the resulting matrix will stay orthogonal if the condition in Equation (8) is met, i.e.,

$$
\left(v_{2}+e^{j \theta_{2}} w_{2}\right)^{*}\left(v_{1}+e^{j \theta_{1}} w_{1}\right)=0 .
$$

Let $\epsilon>0$ be the measure for orthogonality that the system can bear. We can then say that the columns of the resulting matrix are orthogonal up-to $\epsilon$ error, if

$$
\frac{\left|\left(v_{2}+e^{j \theta_{2}} w_{2}\right)^{*}\left(v_{1}+e^{j \theta_{1}} w_{1}\right)\right|^{2}}{\left\|v_{2}+e^{j \theta_{2}} w_{2}\right\|^{2} \cdot\left\|v_{1}+e^{j \theta_{1}} w_{1}\right\|^{2}}<\epsilon .
$$


Let us decompose $e^{j \theta_{1}} w_{1}=u_{1}+z_{1}$ and $e^{j \theta_{2}} w_{2}=u_{2}+z_{2}$, where $u_{1}, u_{2} \in \operatorname{Span}\left(v_{1}, v_{2}\right)$ and $z_{1}, z_{2} \perp \operatorname{Span}\left(v_{1}, v_{2}\right)$. Thus,

$$
\begin{aligned}
& \left(v_{2}+e^{j \theta_{2}} w_{2}\right)^{*}\left(v_{1}+e^{j \theta_{1}} w_{1}\right) \\
& =\left(v_{2}+u_{2}+z_{2}\right)^{*}\left(v_{1}+u_{1}+z_{1}\right) \\
& =\left(v_{2}+u_{2}\right)^{*}\left(v_{1}+u_{1}\right)+z_{2}^{*} z_{1},
\end{aligned}
$$

and

$$
\left\{\begin{array}{l}
\left\|v_{1}+e^{j \theta_{1}} w_{1}\right\|^{2}=\left\|v_{1}+u_{1}\right\|^{2}+\left\|z_{1}\right\|^{2} \\
\left\|v_{2}+e^{j \theta_{2}} w_{2}\right\|^{2}=\left\|v_{2}+u_{2}\right\|^{2}+\left\|z_{2}\right\|^{2} .
\end{array}\right.
$$

Let $\frac{\left|\left(v_{2}+u_{2}\right)^{*}\left(v_{1}+u_{1}\right)\right|}{\left\|v_{2}+u_{2}\right\| \cdot\left\|v_{1}+u_{1}\right\|}=\cos (\varphi)$ and $\frac{\left|z_{2}^{*} z_{1}\right|}{\left\|z_{2}\right\| \cdot\left\|z_{1}\right\|}=\cos (\psi)$, where $\varphi, \psi \in\left[-\frac{\pi}{2}, \frac{\pi}{2}\right]$.

Thus, since $\left\|v_{1}+u_{1}\right\| \cdot\left\|z_{1}\right\| \leq\left\|v_{1}+u_{1}\right\|^{2}+\left\|z_{1}\right\|^{2}$ and $\left\|v_{2}+u_{2}\right\| \cdot\left\|z_{2}\right\| \leq\left\|v_{2}+u_{2}\right\|^{2}+$ $\left\|z_{2}\right\|^{2}$, it follows that

$$
\begin{aligned}
& \frac{\left|\left(v_{2}+e^{j \theta_{2}} w_{2}\right)^{*}\left(v_{1}+e^{j \theta_{1}} w_{1}\right)\right|^{2}}{\left\|v_{2}+e^{j \theta_{2}} w_{2}\right\|^{2} \cdot\left\|v_{1}+e^{j \theta_{1}} w_{1}\right\|^{2}} \\
&=\frac{\left|\left(v_{2}+u_{2}\right)^{*}\left(v_{1}+u_{1}\right)+z_{2}^{*} z_{1}\right|^{2}}{\left(\left\|v_{2}+u_{2}\right\|^{2}+\left\|z_{2}\right\|^{2}\right) \cdot\left(\left\|v_{1}+u_{1}\right\|^{2}+\left\|z_{1}\right\|^{2}\right)} \\
& \leq \frac{\left|\left(v_{2}+u_{2}\right)^{*}\left(v_{1}+u_{1}\right)\right|^{2}+2\left|\left(v_{2}+u_{2}\right)^{*}\left(v_{1}+u_{1}\right)\right|\left|z_{2}^{*} z_{1}\right|+\left|z_{2}^{*} z_{1}\right|^{2}}{\left(\left\|v_{2}+u_{2}\right\|^{2}+\left\|z_{2}\right\|^{2}\right) \cdot\left(\left\|v_{1}+u_{1}\right\|^{2}+\left\|z_{1}\right\|^{2}\right)} \\
& \leq \frac{\left|\left(v_{2}+u_{2}\right)^{*}\left(v_{1}+u_{1}\right)\right|^{2}}{\left\|v_{2}+u_{2}\right\|^{2} \cdot\left\|v_{1}+u_{1}\right\|^{2}}+ \\
& \quad+2 \frac{\left|\left(v_{2}+u_{2}\right)^{*}\left(v_{1}+u_{1}\right)\right|\left|z_{2}^{*} z_{1}\right|}{\left(\left\|v_{2}+u_{2}\right\|^{2}+\left\|z_{2}\right\|^{2}\right) \cdot\left(\left\|v_{1}+u_{1}\right\|^{2}+\left\|z_{1}\right\|^{2}\right)}+ \\
& \quad+\frac{\left|z_{2}^{*} z_{1}\right|^{2}}{\left\|z_{2}\right\|^{2} \cdot\left\|z_{2}\right\|^{2}} \\
& \leq \quad \cos (\varphi)+2 \cos (\varphi) \cos (\psi)+\cos ^{2}(\psi) \\
&=\left(\cos ^{2}(\varphi)+\cos (\psi)\right)^{2} .
\end{aligned}
$$

Now, assume that $\varphi, \psi \in\left[-\frac{\pi}{2},-\frac{\pi}{2}+\delta\right]$ or $\varphi, \psi \in\left[\frac{\pi}{2}-\delta, \frac{\pi}{2}\right]$ for some small $\delta>0$. Thus, $\cos (\varphi), \cos (\psi) \leq \sin (\delta) \leq \delta$, implying that $(\cos (\varphi)+\cos (\psi))^{2} \leq 4 \delta^{2}$. Therefore, for Equation (A2) to hold, it must be the case that $4 \delta^{2}<\epsilon$, or $\delta<\frac{\sqrt{\epsilon}}{2}$.

Theorem A1. Let $0<\epsilon<1$ be the distance from orthogonality that the system allows. In the notions given above, assume that $\varphi, \psi$ are uniformly distributed in $\left[-\frac{\pi}{2}, \frac{\pi}{2}\right] \times\left[-\frac{\pi}{2}, \frac{\pi}{2}\right]$. Thus, the probability that the channel response matrix of the signal received vector, combined from the transmitter and the jammer links, has orthogonal columns up to $\epsilon$, which is less than $\frac{\epsilon}{\pi^{2}}$. 
Proof of Theorem 1. Let $\delta<\frac{\sqrt{\epsilon}}{2}$. Therefore,

$$
\begin{aligned}
& P\left(\frac{\left|\left(v_{2}+e^{j \alpha_{1,2}} w_{1}+e^{j \alpha_{2,2}} w_{2}\right)^{*}\left(v_{1}+e^{j \alpha_{1,1}} w_{1}+e^{j \alpha_{2,1}} w_{2}\right)\right|^{2}}{\left\|v_{2}+e^{j \alpha_{1,2}} w_{1}+e^{j \alpha_{2,2}} w_{2}\right\|^{2} \cdot\left\|v_{1}+e^{j \alpha_{1,1}} w_{1}+e^{j \alpha_{2,1}} w_{2}\right\|^{2}}<\epsilon\right) \\
& \leq P\left((\cos (\varphi)+\cos (\psi))^{2}<\epsilon\right) \\
& \leq \frac{4 \delta^{2}}{\pi^{2}}<\frac{\epsilon}{\pi^{2}} .
\end{aligned}
$$

In order to illustrate the result of the theorem, assume that the interference is acting by exchanging the UE channel-matrix columns, i.e., $e^{j \alpha_{1,2}} w_{1}+e^{j \alpha_{2,2}} w_{2}=v_{1}$ and $e^{j \alpha_{1,1}} w_{1}+$ $e^{j \alpha_{2,1}} w_{2}=v_{2}$. We then obtain

$$
\begin{aligned}
& \frac{\left|\left(v_{2}+e^{j \alpha_{1,2}} w_{1}+e^{j \alpha_{2,2}} w_{2}\right)^{*}\left(v_{1}+e^{j \alpha_{1,1}} w_{1}+e^{j \alpha_{2,1}} w_{2}\right)\right|^{2}}{\left\|v_{2}+e^{j \alpha_{1,2}} w_{1}+e^{j \alpha_{2,2}} w_{2}\right\|^{2} \cdot\left\|v_{1}+e^{j \alpha_{1,1}} w_{1}+e^{j \alpha_{2,1}} w_{2}\right\|^{2}} \\
& =\frac{\left|\left(v_{2}+v_{1}\right)^{*}\left(v_{1}+v_{2}\right)\right|^{2}}{\left\|v_{2}+v_{1}\right\|^{2} \cdot\left\|v_{1}+v_{2}\right\|^{2}} \\
& =\frac{\left(\left\|v_{2}\right\|^{2}+\left\|v_{1}\right\|^{2}\right)^{2}}{\left(\left\|v_{2}\right\|^{2}+\left\|v_{1}\right\|^{2}\right) \cdot\left(\left\|v_{1}\right\|^{2}+\left\|v_{2}\right\|^{2}\right)}=1,
\end{aligned}
$$

which is the worst possible case, and the columns of the resulting combined matrix cannot be accepted as orthogonal in any reasonable system.

\section{References}

1. Alamouti, S.M. A simple transmit diversity technique for wireless communications. IEEE J. Sel. Areas Commun. 1998, 16, 1451-1458. [CrossRef]

2. Zaidi, A.; Athley, F.; Medbo, J.; Gustavsson, U.; Durisi, G.; Chen, X. 5G Physical Layer: Principles, Models and Technology Components; Academic Press: Cambridge, MA, USA, 2018.

3. Chelli, A.; Patzold, M. A MIMO mobile-to-mobile channel model derived from a geometric street scattering model. In Proceedings of the 2007 4th International Symposium on Wireless Communication Systems, Trondheim, Norway, 17-19 October 2007; pp. 792-797.

4. Hogstad, B.O.; Patzold, M. Capacity studies of MIMO channel models based on the geometrical one-ring scattering model. In Proceedings of the 2004 IEEE 15th International Symposium on Personal, Indoor and Mobile Radio Communications (IEEE Cat. No. 04TH8754), Barcelona, Spain, 5-8 September 2004; Volume 3, pp. 1613-1617.

5. Pätzold, M.; Hogstad, B.O. Design and performance of MIMO channel simulators derived from the two-ring scattering model. In Proceedings of the 14th IST Mobile \& Communications Summit, IST 2005, Dresden, Germany, 19-23 June 2005.

6. Almers, P.; Bonek, E.; Burr, A.; Czink, N.; Debbah, M.; Degli-Esposti, V.; Hofstetter, H.; Kyösti, P.; Laurenson, D.; Matz, G.; et al. Survey of channel and radio propagation models for wireless MIMO systems. EURASIP J. Wirel. Commun. Netw. 2007, 2007, 019070. [CrossRef]

7. Saito, Y.; Kishiyama, Y.; Benjebbour, A.; Nakamura, T.; Li, A.; Higuchi, K. Non-orthogonal multiple access (NOMA) for cellular future radio access. In Proceedings of the 14th 2013 IEEE 77th Vehicular Technology Conference (VTC Spring), Dresden, Germany, 2-5 June 2013 ; pp. 1-5.

8. Higuchi, K.; Benjebbour, A. Non-orthogonal multiple access (NOMA) with successive interference cancellation for future radio access. IEICE Trans. Commun. 2015, 98, 403-414. [CrossRef]

9. Singh, K.; Wang, K.; Biswas, S.; Ding, Z.; Khan, F.A.; Ratnarajah, T. Resource optimization in full duplex non-orthogonal multiple access systems. IEEE Trans. Wirel. Commun. 2019, 18, 4312-4325. [CrossRef]

10. Duplicy, J.; Badic, B.; Balraj, R.; Ghaffar, R.; Horváth, P.; Kaltenberger, F.; Knopp, R.; Kovács, I.Z.; Nguyen, H.T.; Tandur, D.; et al. Mu-mimo in lte systems. EURASIP J. Wirel. Commun. Netw. 2011, 2011, 1-13. [CrossRef]

11. Kim, B.H.; Malladi, D.P. Approach to a Unified SU-MIMO/MU-MIMO Operation. U.S. Patent 8,271,043, 18 September 2012.

12. Koc, A.; Masmoudi, A.; Le-Ngoc, T. 3D angular-based hybrid precoding and user grouping for uniform rectangular arrays in massive MU-MIMO systems. IEEE Access 2020, 8, 84689-84712. [CrossRef] 
13. Yang, S.; Chen, P.; She, X.; Liang, L.; Yang, B. Interference modeling and performance evaluation for BS MMSE-IRC receiver in LTE-A release 13. In Proceedings of the 2016 19th International Symposium on Wireless Personal Multimedia Communications (WPMC), Shenzhen, China, 14-16 November 2016; pp. 239-243.

14. Zeng, Z.; Xiao, D.; Han, X. Adaptive IRC algorithm to overcome inter-cell interference in uplink LTE system. In Proceedings of the 2011 International Conference on Multimedia Technology, Hangzhou, China, 26-28 July 2011; pp. 2914-2917.

15. Vijayarani, R.; Nithyanandan, L. Selective Interference Rejection based Antenna Selection for MIMO over LTE Advanced Networks. Procedia Comput. Sci. 2016, 93, 624-631. [CrossRef]

16. Mukherjee, A.; Cheng, J.F.; Falahati, S.; Koorapaty, H.; Karaki, R.; Falconetti, L.; Larsson, D. Licensed-assisted access LTE: Coexistence with IEEE 802.11 and the evolution toward 5G. IEEE Commun. Mag. 2016, 54, 50-57. [CrossRef]

17. Gao, Y. LTE-LAA and WiFi in 5G NR Unlicensed: Fairness, Optimization and Win-Win Solution. In Proceedings of the 2019 IEEE SmartWorld, Ubiquitous Intelligence \& Computing, Advanced \& Trusted Computing, Scalable Computing \& Communications, Cloud \& Big Data Computing, Internet of People and Smart City Innovation (SmartWorld/SCALCOM/UIC/ATC/CBDCom/IOP/SCI), Leicester, UK, 19-23 August 2019; pp. 1638-1643.

18. Doppler, K.; Rinne, M.; Wijting, C.; Ribeiro, C.B.; Hugl, K. Device-to-device communication as an underlay to LTE-advanced networks. IEEE Commun. Mag. 2009, 47, 42-49. [CrossRef]

19. Sichitiu, M.L.; Kihl, M. Inter-vehicle communication systems: A survey. IEEE Commun. Surv. Tutor. 2008, 10, 88-105. [CrossRef]

20. Ezri, D.; Tsodik, G. The impact of synchronization on receive beamforming with null steering in ofdm mimo systems. In Proceedings of the 2012 IEEE 27th Convention of Electrical and Electronics Engineers in Israel, Eilat, Israel, 14-17 November 2012; pp. 1-4.

21. Mahal, J.A. Analysis of Jamming-Vulnerabilities of Modern Multi-carrier Communication Systems. Ph.D. Thesis, Virginia Tech, Arlington, Virginia, 2018.

22. Poisel, R. Modern Communications Jamming Principles and Techniques; Artech House: Boston, MA, USA; London, UK, 2011.

23. Huo, Y.; Tian, Y.; Ma, L.; Cheng, X.; Jing, T. Jamming strategies for physical layer security. IEEE Wirel. Commun. 2017, 25, 148-153. [CrossRef]

24. Slusar, M.V. Driving Analysis Using Vehicle-to-Vehicle Communication. U.S. Patent 9,147,353, 29 September 2015.

25. Marzetta, T.L. Fundamentals of Massive MIMO; Cambridge University Press: Cambridge, UK, 2016.

26. Björnson, E.; Sanguinetti, L.; Wymeersch, H.; Hoydis, J.; Marzetta, T.L. Massive MIMO is a reality What is next? Five promising research directions for antenna arrays. Digit. Signal Process. 2019, 94, 3-20.

27. Hampton, J.R. Introduction to MIMO Communications; Cambridge University Press: Cambridge, UK, 2013.

28. Gkonis, P.K.; Kaklamani, D.I. Reduced Complexity BER Calculations in Large Scale Spatial Multiplexing Multi-User MIMO Orientations in Frequency Selective Fading Environments. Electronics 2019, 8, 727. [CrossRef]

29. Tuncer, T.E.; Friedlander, B. Classical and Modern Direction-of-Arrival Estimation; Academic Press: Cambridge, MA, USA, 2009.

30. Abu-Rgheff, M.A. 5G Physical Layer Technologies; John Wiley \& Sons: Hoboken, NJ, USA, 2019.

31. Esswie, A.A.; Pedersen, K.I. Cross-Link Interference Suppression By Orthogonal Projector For 5G Dynamic TDD URLLC Systems. arXiv 2020, arXiv:2001.05705.

32. Zafar, B.; Gherekhloo, S.; Roemer, F.; Haardt, M. Impact of synchronization errors on Alamouti-STBC-based cooperative MIMO schemes. In Proceedings of the 2012 IEEE 7th Sensor Array and Multichannel Signal Processing Workshop (SAM), Hoboken, NJ, USA, 17-20 June 2012; pp. 81-84.

33. Wanis, M.M.; Shaheen, E.M.; Samir, M. The Impact of Different Jamming Techniques on OFDM Communication Systems. In Proceedings of the International Conference on Electrical Engineering, San Francisco, CA, USA, 23-25 October 2018; Volume 11, pp. 1-14.

34. Ponos, Z.; Dukic, M.L. Pulse jamming of GPS receiver. In Proceedings of the 4th International Conference on Telecommunications in Modern Satellite, Cable and Broadcasting Services, TELSIKS'99 (Cat. No. 99EX365), Nis, Yugoslavia, 13-15 October 1999; Volume 2, pp. 415-418.

35. Chang, J.W.; Kim, S.J.; Kim, J.W.; Sung, W.J. An Adaptive Adjacent Cell Interference Mitigation Method for Eigen-Beamforming Transmission in Downlink Cellular Systems. J. Korean Inst. Electromagn. Eng. Sci. 2009, 20, 248-256. [CrossRef]

36. Tavares, F.M.; Berardinelli, G.; Mahmood, N.H.; Sorensen, T.B.; Mogensen, P. On the potential of interference rejection combining in B4G networks. In Proceedings of the 2013 IEEE 78th Vehicular Technology Conference (VTC Fall), Las Vegas, NV, USA, 2-5 September 2013; pp. 1-5.

37. Mahmood, N.H.; Garcia, L.G.U.; Popovski, P.; Mogensen, P.E. On the performance of successive interference cancellation in 5G small cell networks. In Proceedings of the 2014 IEEE Wireless Communications and Networking Conference (WCNC), Istanbul, Turkey, 6-9 April 2014; pp. 1154-1159.

38. V13.1.0, E.T. Technical Specification Group Radio Access Network; Performance requirements of MMSE-IRC receiver for LTE BS (3GPP TS 36.884 version 13.1.0 Release 13). Available online: https://portal.3gpp.org/desktopmodules/Specifications/ SpecificationDetails.aspx?specificationId=2923 (accessed on 18 March 2021).

39. Okasaka, S.; Weiler, R.J.; Keusgen, W.; Pudeyev, A.; Maltsev, A.; Karls, I.; Sakaguchi, K. Proof-of-concept of a millimeter-wave integrated heterogeneous network for 5G cellular. Sensors 2016, 16, 1362. [CrossRef] [PubMed]

40. Lopez-Perez, D.; Guvenc, I.; De la Roche, G.; Kountouris, M.; Quek, T.Q.; Zhang, J. Enhanced intercell interference coordination challenges in heterogeneous networks. IEEE Wirel. Commun. 2011, 18, 22-30. [CrossRef] 
41. Raghavan, A.; Gebara, E.; Tentzeris, E.M.; Laskar, J. Analysis and design of an interference canceller for collocated radios. IEEE Trans. Microw. Theory Tech. 2005, 53, 3498-3508. [CrossRef]

42. El Bamby, M.S.; Bennis, M.; Latva-aho, M. UL/DL decoupled user association in dynamic TDD small cell networks. In Proceedings of the 2015 International Symposium on Wireless Communication Systems (ISWCS), Brussels, Belgium, 25-28 August 2015; pp. 456-460.

43. Kim, D.; Kim, D.; Shin, D.; Shin, D.; Kim, Y.H. Cyber Battle Damage Assessment Framework and Detection of Unauthorized Wireless Access Point Using Machine Learning. In Frontier Computing; Hung, J., Yen, N., Hui, L., Eds.; Springer: Singapore, 2019; pp. 510-519.

44. Liang, J.J.; Jeng, L.D.; Wang, C.H. A new partial-band noise jamming model for frequency-hopped MFSK systems. In Proceedings of the 2005 2nd International Symposium on Wireless Communication Systems, Siena, Italy, 5-7 September 2005; pp. 200-204.

45. Chen, J.C.; Wen, C.K.; Wong, K.K. Improved constant envelope multiuser precoding for massive MIMO systems. IEEE Commun. Lett. 2014, 18, 1311-1314. [CrossRef]

46. V15.2.0, E.T. 5G; NR; Physical Layer Procedures for Data. Available online: https://portal.3gpp.org/desktopmodules/ Specifications/SpecificationDetails.aspx?specificationId=3216 (accessed on 18 March 2021). 